\title{
Methylation-associated silencing of TU3A in human cancers
}

\author{
YASUO AWAKURA, EIJIRO NAKAMURA, NORIYUKI ITO, TOSHIYUKI KAMOTO and OSAMU OGAWA \\ Department of Urology, Kyoto University Graduate School of Medicine, Kyoto, Japan
}

Received April 15, 2008; Accepted June 18, 2008

DOI: 10.3892/ijo_00000078

\begin{abstract}
TU3A, located on 3p21.1, was originally identified as a candidate tumor suppressor gene in renal cell carcinoma (RCC). Recently, down-regulation of TU3A expression has been reported not only in RCC but also in other types of cancers. However, no studies have evaluated the mechanism underlying TU3A inactivation. In the present study, we first examined the expression and promoter $\mathrm{CpG}$ island methylation of TU3A in RCC. TU3A mRNA was slightly or not expressed in $3 \mathrm{RCC}$ cell lines (ACHN, Caki-1 and NC65). Bisulfite sequencing of the TU3A promoter and treatment of the RCC cell lines with 5-aza-2'-deoxycytidine and/or trichostatin A revealed an association between TU3A expression and promoter hypermethylation. Next, we analyzed TU3A methylation in primary RCC by using combined bisulfite restriction analysis. Mean methylated fraction was $19.2 \%$ (range: $0-57.3 \%$ ) in 53 conventional RCCs and $2.3 \%$ (range: $0-12.7 \%$ ) in 24 corresponding normal kidneys. We defined a methylation fraction of $>20 \%$ as hypermethylation. TU3A hypermethylation was detected in $22(41.5 \%)$ of 53 RCCs and significantly associated with advanced tumor stage ( $>\mathrm{T} 2$ vs. $\mathrm{T} 1$ and $\mathrm{T} 2$ : $\mathrm{P}=0.005, \geq \mathrm{N} 1$ or M1 vs. N0M0: $\mathrm{P}=0.001$ ) and poor disease-specific survival $(\mathrm{P}=0.0038)$. Furthermore, we observed promoter hypermethylation of TU3A in several types of cancer cell lines and primary cancers of the bladder and testis. To our knowledge, the present study is the first to demonstrate the epigenetic inactivation of TU3A in human cancers. The findings of this study warrant further study to investigate the role of TU3A methylation in cancer development.
\end{abstract}

\section{Introduction}

Renal cell carcinoma (RCC) comprises a heterogeneous group of tumors and is classified into various subtypes according to not only morphological features but also commonly observed genetic abnormalities (1). Conventional RCC is the major

Correspondence to: Dr Osamu Ogawa, Department of Urology, Kyoto University Graduate School of Medicine, 54 Kawahara-cho, Shogoin, Sakyo-ku, Kyoto 606-8507, Japan

E-mail:ogawao@kuhp.kyoto-u.ac.jp

Key words: DNA methylation, renal cell carcinoma, cancer, TU3A subtype of RCC and accounts for approximately $75 \%$ of kidney cancers. Allelic loss at chromosome $3 p$ is an early genetic change in conventional RCC $(2,3)$. Von Hippel-Lindau (VHL) gene was identified on chromosome 3p.25 as the causative gene of VHL syndrome, a rare autosomal dominant familial syndrome consisting of different type of cancers including conventional RCC (3). However, mutation and hypermethylation of this gene has been reported in only approximately $50 \%$ of conventional RCC cases that occur sporadically (3-5). This suggests that chromosome $3 \mathrm{p}$ may contain multiple tumor suppressor genes involved in RCC development. Recently, epigenetic inactivation of 3p suppressor genes including RASSF1A and BLU has been reported in RCC (6-8).

TU3A is a candidate tumor suppressor gene located on 3p21.1. Yamato et al originally identified TU3A as a novel gene on a region of common deletion in RCC (9). Wang et al independently reported this gene as DRR1 (down-regulated in renal cell carcinoma 1) and demonstrated its growthsuppressing activity in TU3A-deficient cancer cell lines by transfecting TU3A-expression vectors (10). In their studies, TU3A mutation was not detected and its expression was absent or remarkably reduced in cell lines and clinical samples of RCC. They suggested that some epigenetic change might induce TU3A silencing in RCC. In addition, recent studies have reported the decreased expression of TU3A in a variety of cancers (11-15). To date, no studies have confirmed the epigenetic inactivation of TU3A in human cancers. Herein, we show for the first time methylation-associated silencing of TU3A in RCC. Furthermore, we investigated the methylation status and expression of TU3A in other types of cancers.

\section{Materials and methods}

Clinical samples. We obtained 111 tumor specimens and 24 corresponding normal renal tissues from 53 patients with conventional RCC, 8 patients with papillary RCC, 44 patients with bladder cancer, and 6 patients with testicular cancer who had undergone surgery at Kyoto University Hospital. The tumors were staged according to the International TNM classification system and were graded based on the Fuhrman grading system for RCC (16) and WHO criteria for bladder cancer. Each tissue specimen was snap-frozen immediately after surgical resection and was kept at $-80^{\circ} \mathrm{C}$ until DNA extraction. The institutional review board of the Kyoto University Graduate School of Medicine approved the present study. 
Cell lines. A total of 17 cancer cell lines were analyzed, including 3 RCC cell lines (Caki-1, ACHN and NC65), 8 bladder cancer cell lines (KU7, T24, HT1197, HT1376, J82, UMUC3, 293J and EJ), 1 testicular cancer cell line (NEC3), 1 ovarian cancer cell line (SKOV3), 1 breast cancer cell line (MCF7), 1 cervical cancer cell line (HeLa), 1 gastric cancer cell line (KATO-III) and 1 hepatic cell carcinoma cell line (HepG2). NC65 was kindly gifted by Dr F.H. Schroder (Erasmus University MC Rotterdam, The Netherlands). One (KU7) and 3 (HT1376, UMUC3, and 293J) bladder cancer cell lines were kindly provided by Dr M. Murai (Keio University, Japan) and Dr M.A. Knowles (St. James's University Hospital, UK), respectively. The other cell lines were purchased from American Type Culture Collection (Manassas, VA) or Health Science Research Resources Bank (Tokyo, Japan). All the cell lines were maintained based on the provider's recommendations.

$R N A$ isolation and reverse transcription- $P C R(R T-P C R)$. Total RNA was isolated using RNeasy Kit and RNase-Free DNase (Qiagen, Valencia, CA) according to the manufacturer's protocol. RNA integrity was verified by using the Agilent Bioanalyzer (Agilent Technologies, Palo Alto, CA). Reverse transcription was performed using $3 \mu \mathrm{g}$ of total RNA and First-Strand cDNA Synthesis kit (Amersham Biosciences, Piscataway, NJ). The primer sequences used were 5'-TGG AAT CCG GAG CTC ATC AA-3' and 5'-GCT GGT CAG TGT GGC AAT TCT-3' for TU3A, 5'-TGA ACA GTT GTG GAA CTG TGG-3' and 5'-TAC AGA AGG GCT GAA GGA GG-3' for TU3A transcript variant 1 (TU3Av1), 5'-AGT GCT GGC TCT GCA GTA GGA-3' and 5'-TTC CAG CTC CTC CTT CTT CTT-3' for TU3Av3, and 5'-TAC CTG GTT GAT CCT GCC AGT-3' and 5'-ATC TAG AGT CAC CAA AGC CGC-3' for 18S rRNA.

Rapid amplification of cDNA ends (RACE) analysis. 5'-RACE analysis was performed using FirstChoice ${ }^{\circledR}$ RLM-RACE kit (Ambion, Austin, TX) according to the company's protocol. The normal kidney cDNA was used for nested amplification of TU3A 5'-end. The TU3A-specific primers were 5'-CTC CCT CTG GAT CTC CGA GTA-3' for the first PCR and 5'-TCT GGA TCT CCG AGT ACA TGG C-3' for the second PCR. Amplified PCR products were cloned into pCR2.1 vector using TOPO TA cloning kit (Invitrogen, Carlsbad, CA). Ten clones were randomly selected and sequenced using Big-Dye Terminator Cycle Sequencing Ready Reaction Kit (Applied Biosystems, Foster City, CA) with an ABI PRISM 310 Genetic Analyzer (Applied Biosystems).

Demethylation and inhibition of histone deacetylation. Three RCC cell lines (ACHN, Caki-1, and NC65) were split to low density in $10-\mathrm{cm}$ dishes and grown for $24 \mathrm{~h}$. They were then treated with: i) $10 \mu \mathrm{M}$ 5-aza-2'-deoxycytidine (DAC; Sigma, St. Louis, MO) for $72 \mathrm{~h}$, ii) $10 \mu \mathrm{M}$ DAC for $72 \mathrm{~h}$ followed by $300 \mathrm{nM}$ Trichostatin A (TSA; Wako, Osaka, Japan) for an additional $24 \mathrm{~h}$ or iii) $300 \mathrm{nM}$ TSA for $24 \mathrm{~h}$. The medium containing DAC was replaced every $24 \mathrm{~h}$.

DNA extraction, bisulfite modification and sequencing. Genomic DNA was extracted as described previously (17) or with QIAmp DNA mini kit (Qiagen). DNA was treated with sodium bisulfite using Epitect Bisulfite kit (Qiagen) according to the manufacturer's protocol.

The 2 sequences (333 and $323 \mathrm{bp}$ ), which cover TU3A putative promoter regions (P1 and P2), were amplified from cancer cell lines and primary samples. The primer sequences were 5'-GTT AAA GAA AGT AGA AGA GTA GGT GGG AGA-3' and 5'-TCC TAC TAC AAA ACC AAC ACT ACT AAC CCC-3' for P1, and 5'-GTT AGT TAT TTT TTG TTT TGG GTA AGG AG-3' and 5'-AAA ACT ACT CCT TCT CTA CCT ATC CC-3' for P2. The amplified products were then cloned into pCR2.1 vector using TOPO TA cloning kit (Invitrogen). The plasmid DNA was purified with QIAprep Spin Mini Prep Kit (Qiagen). Eight clones were sequenced for each sample.

Combined bisulfite restriction analysis (COBRA). PCR was performed with the primer sets for the promoter regions $\mathrm{P} 1$ and P2. PCR products were incubated with restriction enzymes Hpy $188 \mathrm{I}$ for $2 \mathrm{~h}$ at $37^{\circ} \mathrm{C}$ and TaqI for $1 \mathrm{~h}$ at $65^{\circ} \mathrm{C}$ to examine the methylation in promoter regions $\mathrm{P} 1$ and $\mathrm{P} 2$, respectively. The digested products were loaded onto a DNA 500 LabChip (Agilent) and were assayed with the Agilent Bioanalyzer (Agilent). The percentage of methylation in the promoter $\mathrm{CpG}$ island of TU3A was calculated as described previously (18), using the following formula: peak height of methylated products/(peak height of methylated products + peak height of unmethylated product).

Statistical analysis. Fisher's exact test was performed to analyze the relationship between TU3A methylation status and clinical/pathological factors. Disease-specific survival was defined as the time from surgery to death if the patient died of disease, or until the last contact. Disease-specific survival curves were generated by the Kaplan-Meier method, and the difference between the groups defined by TU3A methylation status was compared by the log-rank test. Statistical significance was defined as $\mathrm{P}<0.05$. Statistical analyses were performed using Dr SPSS II (SPSS, Chicago, IL).

\section{Results}

Structure of the TU3A gene. The cDNA sequences of TU3A transcript variants (designated as TU3Av1 and TU3Av2 in the present study) are available in Genbank database (accession no. NM_007177 and NM_001076778, respectively). In addition, Yamato et al previously isolated a splicing variant of TU3A (accession no. AB030702). This variant harbors a 21-bp alternative exon in the 5' untranslated region, which continues into exon 3 of TU3Av1 (9). We confirmed the presence of a 111-bp alternative exon including the 21-bp sequence by 5'-RACE. Subsequently, we sequenced a 2680-bp transcript, which was termed TU3Av3. This sequence is availabale in Genbank database (accession no. EU569833). As shown in Fig. 1, the 3 variants encode the same amino acid sequences but differ in the structure of 5'-untranslated region. RT-PCR revealed the expression of TU3Av3 in the primary normal kidney (Fig. 2).

As shown in Figs. 1 and 2, CpG islands are located $\sim 17-\mathrm{kb}$ and $15-\mathrm{kb}$ upstream of the TU3A translational start site. 


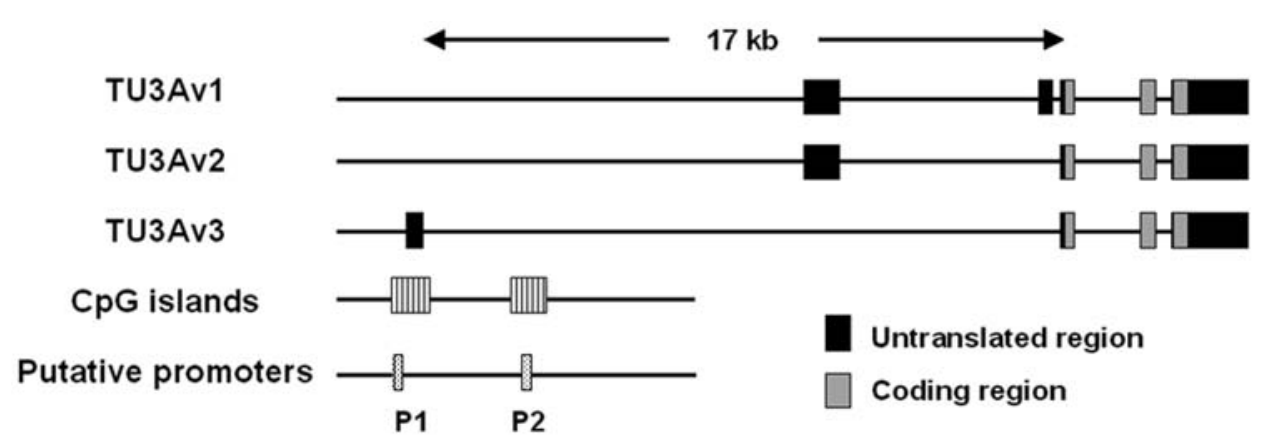

Figure 1. Schematic diagram of the TU3A gene structure.

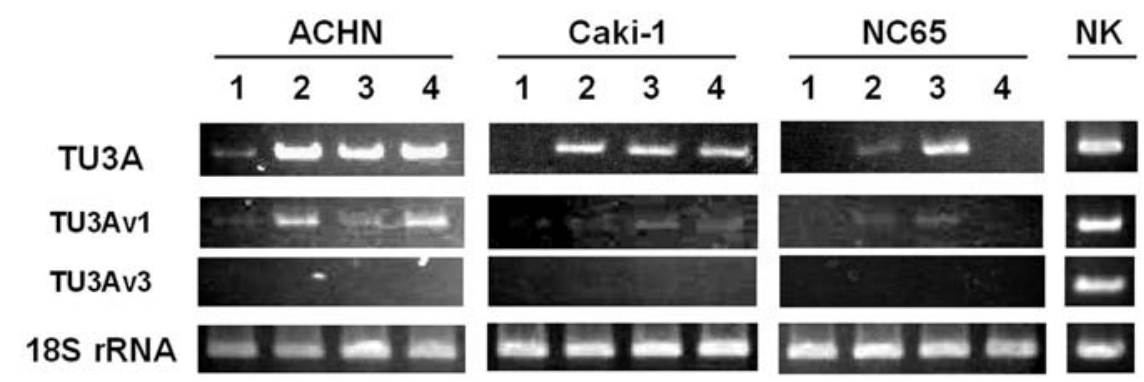

Figure 2. RT-PCR analysis of TU3A in RCC cell lines and normal kidney (NK). The RCC cell lines were subjected to 4 different treatments: Mock (1), DAC (2), DAC + TSA (3), and TSA (4).

Exon 1 of TU3Av3 is within the $\mathrm{CpG}$ islands $17-\mathrm{kb}$ upstream to TU3A translational start site. The 2 TU3A putative promoter regions (designated as $\mathrm{P} 1$ and $\mathrm{P} 2$ ) were predicted from the genomic sequence $20-\mathrm{kb}$ upstream and $10-\mathrm{kb}$ downstream of the presumed TU3Av1 transcriptional start site using the PromoterInspector prediction software (http://www.genomatix.ed) (19). Both P1 and TU3Av3 exon 1 are within the $\mathrm{CpG}$ island $17-\mathrm{kb}$ upstream of TU3A translational start site, and P1 is located upstream of TU3Av3 exon 1. P2 is within the $\mathrm{CpG}$ island $15-\mathrm{kb}$ upstream of TU3A translational start site.

Re-expression of TU3A in RCC cell lines by demethylation and/or inhibition of histone deacetylation. We examined TU3A expression by RT-PCR (Fig. 2). ACHN expressed low levels of TU3A mRNA. Neither Caki-1 nor NC65 expressed TU3A. In ACHN and Caki-1, TU3A mRNA expression was induced after DAC and/or TSA treatment. In NC65, SFRP1 expression was detected after DAC treatment but not TSA treatment. The combination of DAC and TSA remarkably elevated the level of TU3A mRNA expression that had been induced by DAC in NC65.

Next, we performed RT-PCR with the primer sets specific for TU3Av1 or TU3Av3 (Fig. 2). DAC and/or TSA treatment induced TU3Av1 expression in all the 3 cell lines, while none of the 3 cell lines expressed the TU3Av3 transcript after DAC and/or TSA treatment.

Methylation status of TU3A in RCC cell lines. We analyzed the methylation status of the TU3A promoter in the $3 \mathrm{RCC}$ cell lines and peripheral blood leukocytes (PBLs) by bisulfite sequencing and COBRA (Fig. 3). Bisulfite sequencing demonstrated that $\mathrm{P} 1$ region was densely methylated in all the analyzed ACHN, Caki-1 and NC65 clones (Fig. 3A). COBRA of $\mathrm{P} 1$ region revealed $100 \%$ methylated fractions in all the cell lines (Fig. 3B). In contrast, P2 region was not methylated in any of the analyzed ACHN and Caki-1 clones. Of 8 NC65 clones, only 2 were densely methylated (data not shown). COBRA of $\mathrm{P} 2$ region revealed no methylated fraction in ACHN and Caki-1, and a $28.7 \%$ methylation percentage was obtained for NC65 (Fig. 3B). These findings and the result of TU3A re-expression induced by DAC and/or TSA treatment in RCC cell lines suggest the importance of $\mathrm{P} 1$ region for TU3A expression.

Next, we examined the methylation status of TU3A in PBLs from a healthy individual. Bisulfite sequencing and COBRA revealed virtually no methylation in $\mathrm{P} 1$ or $\mathrm{P} 2$ (Fig. 3).

Methylation status of TU3A in primary RCC. We analyzed the methylation status of TU3A promoter $\mathrm{P} 1$ in the 53 primary conventional RCCs and corresponding 24 normal kidney tissues by COBRA (Fig. 4). Methylated and unmethylated clones in bisulfite sequencing were used as controls. The mean methylation percentage was $19.2 \%$ (range: $0-57.3 \%$ ) in conventional RCCs and $2.4 \%$ (range: $0-12.7 \%$ ) in normal kidneys. We defined a methylation fraction of $>20 \%$ as hypermethylation. TU3A was hypermethylated in $22(41.5 \%)$ of 53 conventional RCCs (Table I). Methylation status (hyper-methylated or not) of TU3A was significantly associated with tumor stage ( $>\mathrm{T} 2$ vs. $\mathrm{T} 1$ and $\mathrm{T} 2$ : $\mathrm{P}=0.005$, $\geq \mathrm{N} 1$ or M1 vs. N0M0: $\mathrm{P}=0.001)$, whereas it was not 
A

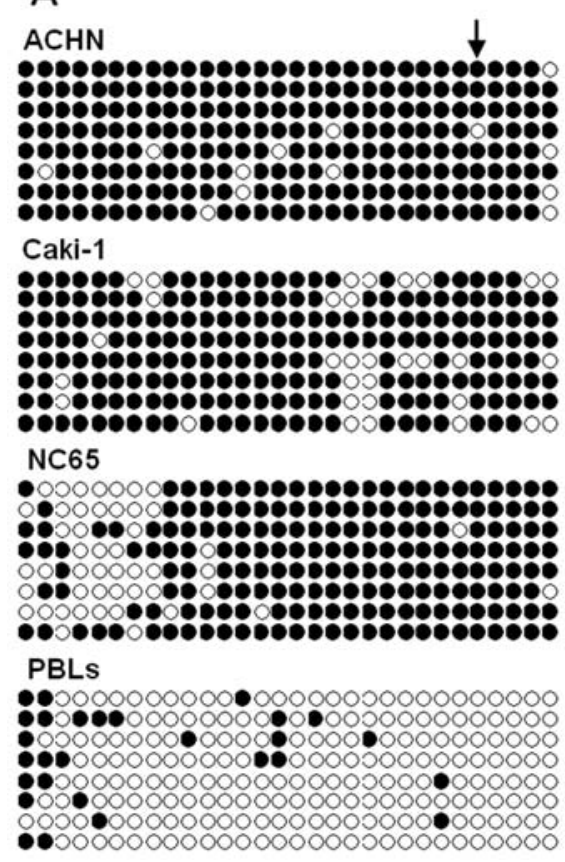

B
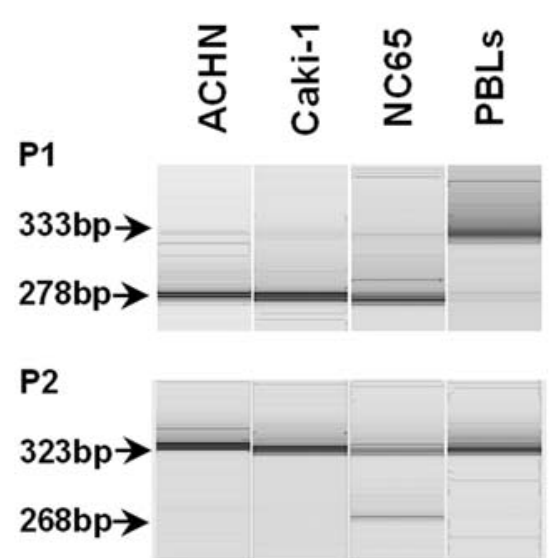

Figure 3. A, Bisulfite sequencing analysis of TU3A promoter P1 in the 3 RCC cell lines (ACHN, Caki-1 and NC65) and PBLs. Open and filled circles represent unmethylated and methylated $\mathrm{CpG}$ sites, respectively, and each row represents a single clone. The arrow shows the recognition site of restriction enzyme Hpy188I. B, COBRA of TU3A promoters P1 and P2 from the same samples.

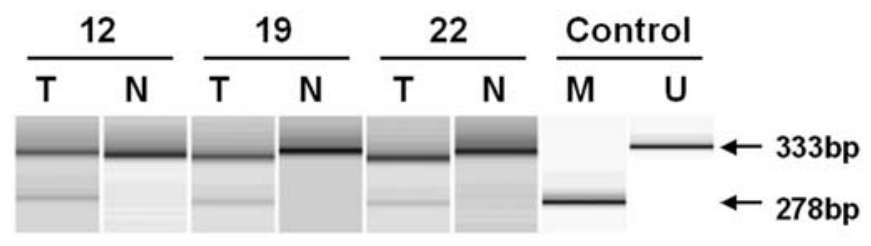

Figure 4. COBRA of TU3A promoter P1 in primary conventional RCC. The number corresponds to that in Table $\mathrm{I}$. T and $\mathrm{N}$ denote tumor and normal kidney, respectively.

significantly related to age, gender or histological grade $(\geq 60$ vs. <60: $\mathrm{P}=1.0$, gender: $\mathrm{P}=1.0, \mathrm{G} 1$ and $\mathrm{G} 2$ vs. $\mathrm{G} 3$ and $\mathrm{G} 4$ : $\mathrm{P}=0.073)$. Furthermore, TU3 A methylation adversely affected disease-specific survival in the patients with conventional RCC (Fig. 5, P=0.0038). TU3A was hypermethylated in $2(25 \%)$ of 8 papillary RCCs.

Expression and methylation status of TU3A in several types of cancer cell lines. Yamato et al and Wang et al have reported that TU3A is ubiquitously expressed in normal tissues except PBLs $(9,10)$. Furthermore, Chen et al have identified TU3A as one of the commonly down-regulated genes in 10 different types of cancer cell lines by using a bioinformatics tool from the UniGene database (20). These findings prompted us to examine the expression and methylation status of TU3A in several cancer cell lines originating from organs other than kidney. RT-PCR revealed no expression in 11 of the 14 cancer cell lines and slight expression in 2 bladder cancer cell lines (UMUC3 and 293J) and a testicular cancer cell line (NEC8) (Fig. 6A). COBRA of promoter P1 revealed virtually $100 \%$ methylation in 8 of 11 cell lines without

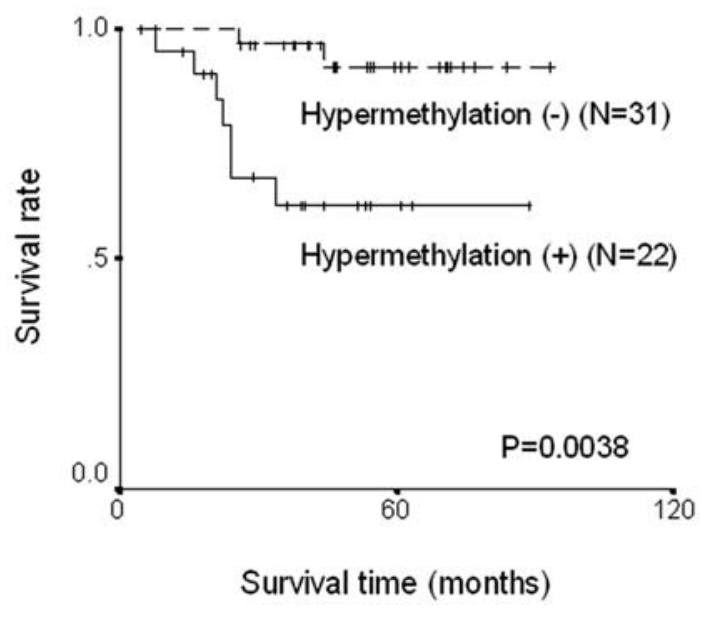

Figure 5. Disease-specific survival according to TU3A methylation status in the patients with conventional RCC.

TU3A expression and 2 cell lines with TU3A slight expression (Fig. 6B). In NEC8, which expressed low levels of TU3A transcript, a $63.2 \%$ methylation fraction was detected. These findings suggest the association between the methylation of promoter P1 and TU3A expression. However, COBRA revealed no or partial methylation (0-54.5\%) in 3 cell lines without TU3A expression (HT1197, HT1376 and SKOV3) (Fig. 6B). In HT1376, TSA treatment restored TU3A expression (data not shown). The epigenetic inactivation of TU3A may occur despite poor methylation of promoter $\mathrm{CpG}$ island in the 3 cell lines (21). COBRA of promoter P2 showed no methylation in any of the cell lines, except KU7 (Fig. 6B). 
Table I. Clinicopathological factors and TU3A methylation status in the patients with conventional RCC.

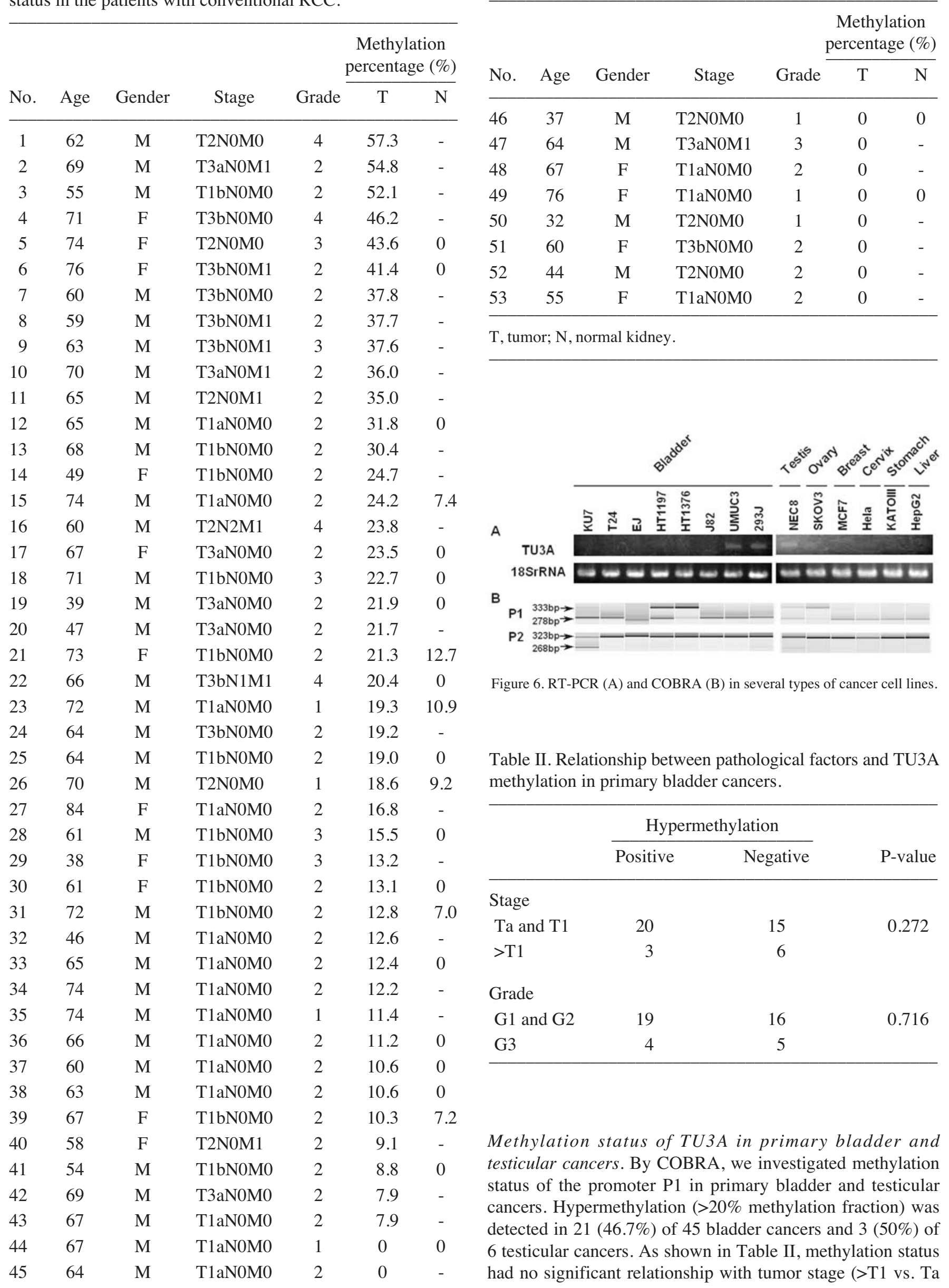

Table I. Continued.

(n)


and T1: $\mathrm{P}=0.272)$ and grade $(\mathrm{G} 1$ and $\mathrm{G} 2$ vs. $\mathrm{G} 3: \mathrm{P}=0.716)$ in bladder cancers.

\section{Discussion}

In the present study, we first demonstrated that TU3A was frequently inactivated by hypermethylation of its promoter in RCC and that the methylation status of TU3A promoter is significantly related to RCC prognosis. RCC is characterized by a variable and unpredictable clinical course and thus, accurate prediction of the prognosis is important in clinical settings (22). It might be informative to evaluate the clinical usefulness of TU3A methylation as a prognostic marker in a large number of RCC patients. Next, we demonstrated TU3A silencing and promoter hypermethylation in other types of cancer. Recently, TU3A has been reported to be down-regulated in primary carcinomas of the prostate $(11,12)$, colon $(13)$ and brain $(14,15)$. In particular, the reduction of TU3A expression has been shown to be associated with progression in prostate cancer (12) and astrocytoma (15). These cancers were not investigated in the present study. The epigenetic silencing of TU3A may also be a frequent phenomenon in these cancers and have an adverse impact on their clinical course.

Spira et al have shown that smoking contributes to the decrease of TU3A expression in the epithelial cells of the pulmonary airway by microarray analysis of clinical samples (23). In the present study, we revealed TU3A inactivation and promoter hypermethylation in lung cancer cell lines. It might be interesting to examine the relationship between TU3A methylation and smoking in lung carcinogenesis.

TU3A gene encodes a protein consisting of 144 amino acids with a nuclear localization signal and a coiled-coil domain. Wang et al experimentally confirmed that TU3A protein was present in the nucleus (10). In addition, coiledcoil domain has been identified in nuclear proteins including a class of transcription factors, the leucine zipper proteins (24). Thus, TU3A may regulate gene expression by interacting with DNA and/or other proteins.

In conclusion, we showed the epigenetic inactivation of TU3A in several types of cancer cell lines and primary cancers. Previous studies reported that TU3A was ubiquitously expressed in normal tissues and frequently down-regulated in a variety of cancers. Therefore, the present results warrant further study in order to investigate the role of TU3A silencing in early carcinogenesis and cancer progression.

\section{Acknowledgements}

This work was supported by a grant from the Ministry of Education, Culture, Sports, Science and Technology, Japan.

\section{References}

1. Bodmer D, van den Hurk W, van Groningen JJ, Eleveld MJ, Martens GJ, Weterman MA and van Kessel AG: Understanding familial and non-familial renal cell cancer. Hum Mol Genet 11: 2489-2498, 2002.

2. Ogawa O, Kakehi Y, Ogawa K, Koshiba M, Sugiyama T and Yoshida O: Allelic loss at chromosome 3p characterizes clear cell phenotype of renal cell carcinoma. Cancer Res 51: 949-953, 1991.
3. Kondo K, Yao M, Yoshida M, Kishida T, Shuin T, Miura T, Moriyama M, Kobayashi K, Sakai N, Kaneko S, Kawakami S, Baba M, Nakaigawa N, Nagashima Y, Nakatani Y and Hosaka M: Comprehensive mutational analysis of the VHL gene in sporadic renal cell carcinoma: relationship to clinicopathological parameters. Genes Chromosomes Cancer 34: 58-68, 2002.

4. Brauch H, Weirich G, Brieger J, Glavac D, Rodl H, Eichinger M, Feurer M, Weidt E, Puranakanitstha C, Neuhaus C, Pomer S, Brenner W, Schirmacher P, Storkel S, Rotter M, Masera A, Gugeler N and Decker HJ: VHL alterations in human clear cell renal cell carcinoma: association with advanced tumor stage and a novel hot-spot mutation. Cancer Res 60: 1942-1948, 2000.

5. Hamano K, Esumi M, Igarashi H, Chino K, Mochida J, Ishida H and Okada K: Biallelic inactivation of the von Hippel-Lindau tumor suppressor gene in sporadic renal cell carcinoma. J Urol 167: 713-717, 2002.

6. Morrissey C, Martinez A, Zatyka M, Agathanggelou A, Honorio S, Astuti D, Morgan NV, Moch H, Richards FM, Kishida T, Yao M, Schraml P, Latif F and Maher ER: Epigenetic inactivation of the RASSF1A 3p21.3 tumor suppressor gene in both clear cell and papillary renal cell carcinoma. Cancer Res 61: 7277-7281, 2001.

7. Dreijerink K, Braga E, Kuzmin I, Geil L, Duh FM, Angeloni D, Zbar B, Lerman MI, Stanbridge EJ, Minna JD, Protopopov A, Li J, Kashuba V, Klein G and Zabarovsky ER: The candidate tumor suppressor gene, RASSF1A, from human chromosome 3p21.3 is involved in kidney tumorigenesis. Proc Natl Acad Sci USA 98: 7504-7509, 2001.

8. Agathanggelou A, Dallol A, Zochbauer-Muller S, Morrissey C, Honorio S, Hesson L, Martinsson T, Fong KM, Kuo MJ, Yuen PW, Maher ER, Minna JD and Latif F: Epigenetic inactivation of the candidate $3 \mathrm{p} 21.3$ suppressor gene BLU in human cancers. Oncogene 22: 1580-1588, 2003.

9. Yamato T, Orikasa K, Fukushige S, Orikasa S and Horii A: Isolation and characterization of the novel gene, TU3A, in a commonly deleted region on $3 \mathrm{p} 14.3 \rightarrow \mathrm{p} 14.2$ in renal cell carcinoma. Cytogenet Cell Genet 87: 291-295, 1999.

10. Wang L, Darling J, Zhang JS, Liu W, Qian J, Bostwick D, Hartmann L, Jenkins R, Bardenhauer W, Schutte J, Opalka B and Smith DI: Loss of expression of the DRR 1 gene at chromosomal segment 3p21.1 in renal cell carcinoma. Genes Chromosomes Cancer 27: 1-10, 2000.

11. Kristiansen G, Pilarsky C, Wissmann C, Kaiser S, Bruemmendorf T, Roepcke S, Dahl E, Hinzmann B, Specht T, Pervan J, Stephan C, Loening S, Dietel M and Rosenthal A: Expression profiling of microdissected matched prostate cancer samples reveals CD166/MEMD and CD24 as new prognostic markers for patient survival. J Pathol 205: 359-376, 2005.

12. Vanaja DK, Ballman KV, Morlan BW, Cheville JC, Neumann RM, Lieber MM, Tindall DJ and Young CY: PDLIM4 repression by hypermethylation as a potential biomarker for prostate cancer. Clin Cancer Res 12: 1128-1136, 2006.

13. Eschrich S, Yang I, Bloom G, Kwong KY, Boulware D, Cantor A, Coppola D, Kruhoffer M, Aaltonen L, Orntoft TF, Quackenbush J and Yeatman TJ: Molecular staging for survival prediction of colorectal cancer patients. J Clin Oncol 23: 3526-3535, 2005.

14. Fathallah-Shaykh HM, Rigen M, Zhao LJ, Bansal K, He B, Engelhard HH, Cerullo L, Roenn KV, Byrne R, Munoz L, Rosseau GL, Glick R, Lichtor T and Di Savino E: Mathematical modeling of noise and discovery of genetic expression classes in gliomas. Oncogene 21: 7164-7174, 2002.

15. Van den Boom J, Wolter M, Blaschke B, Knobbe CB and Reifenberger G: Identification of novel genes associated with astrocytoma progression using suppression subtractive hybridization and real-time reverse transcription-polymerase chain reaction. Int J Cancer 119: 2330-2338, 2006.

16. Goldstein NS: Grading of renal cell carcinoma. Urol Clin North Am 26: 637-642, 1999.

17. Ozdemir E, Kakehi Y, Nakamura E, Kinoshita H, Terachi T, Okada Y and Yoshida O: HLA-DRB $1 * 0101$ and $* 0405$ as protective alleles in Japanese patients with renal cell carcinoma. Cancer Res 57: 742-746, 1997.

18. Brena RM, Auer H, Kornacker K, Hackanson B, Raval A, Byrd JC and Plass C: Accurate quantification of DNA methylation using combined bisulfite restriction analysis coupled with the Agilent 2100 Bioanalyzer platform. Nucleic Acids Res 34: e17, 2006. 
19. Scherf M, Klingenhoff A and Werner T: Highly specific localization of promoter regions in large genomic sequences by PromoterInspector: a novel context analysis approach. J Mol Biol 297: 599-606, 2000.

20. Chen S, Zhu B and Yu L: In silico comparison of gene expression levels in ten human tumor types reveals candidate genes associated with carcinogenesis. Cytogenet Genome Res 112: 53-59, 2006.

21. Zhao W, Soejima H, Higashimoto K, Nakagawachi T, Urano T, Kudo S, Matsukura S, Matsuo S, Joh K and Mukai T: The essential role of histone H3 Lys9 di-methylation and MeCP2 binding in MGMT silencing with poor DNA methylation of the promoter CpG island. J Biochem 137: 431-440, 2005.
22. Shimazui T, Oosterwijk E, Debruyne FM and Schalken JA: Molecular prognostic factor in renal cell carcinoma. Semin Urol Oncol 14: 250-255, 1996

23. Spira A, Beane J, Shah V, Liu G, Schembri F, Yang X, Palma J and Brody JS: Effects of cigarette smoke on the human airway epithelial cell transcriptome. Proc Natl Acad Sci USA 101: 10143-10148, 2004.

24. Lupas A: Coiled coils: new structures and new functions. Trends Biochem Sci 21: 375-382, 1996. 